quatrième série-tome 45 fascicule 6 novembre-décembre 2012

$$
\begin{aligned}
& \text { ANNALES } \\
& \text { SCIENTIFIQUES } \\
& \text { de } \\
& \text { L'ECOLE } \\
& \text { NORMALE } \\
& \text { SUPÉRIEURE }
\end{aligned}
$$

Frédéric BAYART \& Yanick HEURTEAUX

Multifractal analysis of the divergence of Fourier series 
Ann. Scient. Éc. Norm. Sup.

$4^{\text {e }}$ série, t. 45, 2012, p. 927 à 946

\title{
MULTIFRACTAL ANALYSIS OF THE DIVERGENCE OF FOURIER SERIES
}

\author{
BY FRÉDÉRIC BAYART AND YANICK HEURTEAUX
}

\begin{abstract}
A famous theorem of Carleson says that, given any function $f \in L^{p}(\mathbb{T})$, $p \in(1,+\infty)$, its Fourier series $\left(S_{n} f(x)\right)$ converges for almost every $x \in \mathbb{T}$. Beside this property, the series may diverge at some point, without exceeding $O\left(n^{1 / p}\right)$. We define the divergence index at $x$ as the infimum of the positive real numbers $\beta$ such that $S_{n} f(x)=O\left(n^{\beta}\right)$ and we are interested in the size of the exceptional sets $E_{\beta}$, namely the sets of $x \in \mathbb{T}$ with divergence index equal to $\beta$. We show that quasi-all functions in $L^{p}(\mathbb{T})$ have a multifractal behavior with respect to this definition. Precisely, for quasi-all functions in $L^{p}(\mathbb{T})$, for all $\beta \in[0,1 / p], E_{\beta}$ has Hausdorff dimension equal to $1-\beta p$. We also investigate the same problem in $\mathscr{C}(\mathbb{T})$, replacing polynomial divergence by logarithmic divergence. In this context, the results that we get on the size of the exceptional sets are rather surprising.

RÉsumÉ. - Un célèbre théorème de Carleson nous dit que si une fonction $f$ est de puissance $p$-ième intégrable $(p>1)$, sa série de Fourier converge presque partout. D'un autre côté, il peut y avoir des points de divergence. Pour un tel point donné $x$, on peut introduire l'indice de divergence comme étant le plus petit exposant $\beta$ tel que $S_{n} f(x)=O\left(n^{\beta}\right)$. On sait que cet indice est au plus égal à $1 / p$ et on s'intéresse à la dimension des ensembles exceptionnels de points $E_{\beta}$ d'indice de divergence donné $\beta$. Nous montrons que quasi-toute fonction de $L^{p}$ (au sens de Baire) a un comportement multifractal. De façon précise, quasi-sûrement dans $L^{p}$, pour tout $\beta$, la dimension de Hausdorff de $E_{\beta}$ vaut $1-\beta p$. Nous nous intéressons aussi aux fonctions continues pour lesquelles la croissance de $S_{n} f(x)$ est contrôlée par le logarithme de $n$. Là encore un indice de divergence (logarithmique) peut être introduit et nous obtenons des résultats surprenants sur la taille des ensembles exceptionnels.
\end{abstract}

\section{Introduction}

\subsection{Description of the results}

The famous theorem of Carleson and Hunt asserts that, when $f$ belongs to $L^{p}(\mathbb{T})$, $1<p<+\infty$, where $\mathbb{T}=\mathbb{R} / \mathbb{Z}$, the sequence of the partial sums of its Fourier series $\left(S_{n} f(x)\right)_{n \geq 0}$ converges for almost every $x \in \mathbb{T}$. On the other hand, it can diverge at some point. This divergence cannot be too fast since, for any $f \in L^{p}(\mathbb{T})$ and any $x \in \mathbb{T}$, $\left|S_{n} f(x)\right| \leq C_{p} n^{1 / p}\|f\|_{p}$ (see [14] for instance). In view of these results, a natural question 
arises. How big can the sets $F$ be such that $\left|S_{n} f(x)\right|$ grows as fast as possible for every $x \in F$ ? More generally, can we say something on the size of the sets such that $\left|S_{n} f(x)\right|$ behaves like (or as bad as) $n^{\beta}$ for some $\beta \in(0,1 / p]$ ?

To measure the size of subsets of $\mathbb{T}$, we shall use the Hausdorff dimension. Let us recall the relevant definitions (we refer to [5] and to [11] for more on this subject). If $\phi: \mathbb{R}_{+} \rightarrow \mathbb{R}_{+}$ is a nondecreasing continuous function satisfying $\phi(0)=0$ ( $\phi$ is called a dimension function or a gauge function), the $\phi$-Hausdorff outer measure of a set $E \subset \mathbb{R}^{d}$ is

$$
\mathcal{H}^{\phi}(E)=\lim _{\varepsilon \rightarrow 0} \inf _{r \in R_{\varepsilon}(E)} \sum_{B \in r} \phi(|B|),
$$

$R_{\varepsilon}(E)$ being the set of countable coverings of $E$ with balls $B$ of diameter $|B| \leq \varepsilon$. When $\phi_{s}(x)=x^{s}$, we write for short $\mathcal{H}^{s}$ instead of $\mathcal{H}^{\phi_{s}}$. The Hausdorff dimension of a set $E$ is

$$
\operatorname{dim}_{\mathcal{H}}(E):=\sup \left\{s>0 ; \mathcal{H}^{s}(E)>0\right\}=\inf \left\{s>0 ; \mathcal{H}^{s}(E)=0\right\} .
$$

There exist old results measuring the size of sets of points of divergence of Fourier series. For example, in the book of Kahane and Salem ([9]), we can find such results for functions belonging to a Sobolev space "close" to $L^{2}$. The relevant result in our context is due to J-M. Aubry [1].

Theorem 1.1. - Let $f \in L^{p}(\mathbb{T}), 1<p<+\infty$. For $\beta \geq 0$, define

$$
\mathcal{E}(\beta, f)=\left\{x \in \mathbb{T} ; \limsup _{n \rightarrow+\infty} n^{-\beta}\left|S_{n} f(x)\right|>0\right\} .
$$

Then $\operatorname{dim}_{\mathcal{H}}(\mathcal{E}(\beta, f)) \leq 1-\beta p$. Conversely, given a set $E$ such that $\operatorname{dim}_{\mathcal{H}_{\mathcal{H}}}(E)<1-\beta p$, there exists a function $f \in L^{p}(\mathbb{T})$ such that, for any $x \in E, \lim _{\sup _{n \rightarrow+\infty}} n^{-\beta}\left|S_{n} f(x)\right|=+\infty$.

This result motivated us to introduce the notion of divergence index. For a given function $f \in L^{p}(\mathbb{T})$ and a given point $x_{0} \in \mathbb{T}$, we can define the real number $\beta\left(x_{0}\right)$ as the infimum of the non negative real numbers $\beta$ such that $\left|S_{n} f\left(x_{0}\right)\right|=O\left(n^{\beta}\right)$. The real number $\beta\left(x_{0}\right)$ will be called the divergence index of the Fourier series of $f$ at point $x_{0}$. Of course, for any function $f \in L^{p}(\mathbb{T})(1<p<+\infty)$ and any point $x_{0} \in \mathbb{T}, 0 \leq \beta\left(x_{0}\right) \leq 1 / p$. Moreover, Carleson's theorem implies that $\beta\left(x_{0}\right)=0$ almost everywhere and we would like to have precise estimates on the size of the level sets of the function $\beta$. These are defined as

$$
\begin{aligned}
E(\beta, f) & =\{x \in \mathbb{T} ; \beta(x)=\beta\} \\
& =\left\{x \in \mathbb{T} ; \limsup _{n \rightarrow+\infty} \frac{\log \left|S_{n} f(x)\right|}{\log n}=\beta\right\} .
\end{aligned}
$$

We can ask for which values of $\beta$ the sets $E(\beta, f)$ are non-empty. This set of values will be called the domain of definition of the divergence spectrum of $f$. If $\beta$ belongs to the domain of definition of the divergence spectrum, it is also interesting to estimate the Hausdorff dimension of the sets $E(\beta, f)$. The function $\beta \mapsto \operatorname{dim}_{\mathcal{H}}(E(\beta, f))$ will be called the divergence spectrum of the function $f$ (in terms of its Fourier series). By Aubry's result, $\operatorname{dim}_{\mathscr{H}}(E(\beta, f)) \leq 1-\beta p$ and, for any fixed $\beta_{0} \in[0,1 / p)$, for any $\varepsilon>0$, one can find $f \in L^{p}(\mathbb{T})$ such that $\operatorname{dim}_{\mathscr{H}}\left(\bigcup_{\beta_{0} \leq \beta \leq 1 / p} E(\beta, f)\right) \geq 1-\beta_{0} p-\varepsilon$. Our first main result is that a typical function $f \in L^{p}(\mathbb{T})$ satisfies $\operatorname{dim}_{\mathcal{H}}(E(\beta, f))=1-\beta p$ for any $\beta \in[0,1 / p]$. In particular, $f$ has a multifractal behavior with respect to the summation of its Fourier series, 
meaning that the domain of definition of its divergence spectrum contains an interval with non-empty interior.

Theorem 1.2. - Let $1<p<+\infty$.

(1) For all functions $f \in L^{p}(\mathbb{T})$, for any $\beta \in[0,1 / p] \operatorname{dim}_{\mathcal{H}}(E(\beta, f)) \leq 1-\beta p$.

(2) For quasi-all functions $f \in L^{p}(\mathbb{T})$, for any $\beta \in[0,1 / p]$, $\operatorname{dim}_{\mathcal{H}}(E(\beta, f))=1-\beta p$.

The terminology "quasi-all" used here is relative to the Baire category theorem. It means that this property is true for a residual set of functions in $L^{p}(\mathbb{T})$. Theorem 1.2 can be compared with other types of results in multifractal analysis, for example regarding Hölder's regularity (see [7]) or fast points for the Brownian motion (see [12]).

In a second part of the paper, we turn to the case of $\mathscr{C}(\mathbb{T})$, the set of continuous functions on $\mathbb{T}$. In that space, the divergence of Fourier series is controlled by a logarithmic factor. More precisely, if $\left(D_{n}\right)$ is the sequence of the Dirichlet kernels, we know that $\left\|S_{n} f\right\|_{\infty} \leq\left\|D_{n}\right\|_{1}\|f\|_{\infty}$, so that there exists some absolute constant $C>0$ such that $\left\|S_{n} f\right\|_{\infty} \leq C\|f\|_{\infty} \log n$ for any $f \in \mathscr{C}(\mathbb{T})$ and any $n>1$. As before, one can discuss the size of the sets such that $\left|S_{n} f(x)\right|$ behaves badly, namely like $(\log n)^{\beta}, \beta \in[0,1]$. More precisely, mimicking the case of the $L^{p}$ spaces, we introduce, for any $\beta \in[0,1]$ and any $f \in \mathscr{C}(\mathbb{T})$, the following sets:

$$
\mathcal{F}(\beta, f)=\left\{x \in \mathbb{T} ; \limsup _{n \rightarrow+\infty}(\log n)^{-\beta}\left|S_{n} f(x)\right|>0\right\} .
$$

When we try to estimate the size of the sets $\mathcal{F}(\beta, f)$, we observe that the Hausdorff dimension is not sufficiently precise. We need a new family of gauge functions. For $s>0$ and $t \in(0,1]$, we consider

$$
\phi_{s, t}(x)=x^{s} \exp \left[(\log 1 / x)^{1-t}\right] .
$$

It is not difficult to check that $\phi_{s, t}(x) \leq \phi_{s^{\prime}, t^{\prime}}(x)$ for small values of $x$ iff

$$
s>s^{\prime} \text { or }\left(s=s^{\prime} \text { and } t \geq t^{\prime}\right) .
$$

The analogue of Aubry's theorem in this context is

Proposition 1.3. - Let $\beta \in(0,1)$ and $f \in \mathscr{C}(\mathbb{T})$. Then, for any $\gamma>1-\beta$,

$$
\mathcal{H}^{\phi_{1, \gamma}}(\mathcal{F}(\beta, f))=0 \text {. }
$$

Following the $L^{p}$ case, we define for $f \in \mathscr{C}(\mathbb{T})$ and $\beta \in[0,1]$ the level set

$$
F(\beta, f)=\left\{x \in \mathbb{T} ; \limsup _{n \rightarrow+\infty} \frac{\log \left|S_{n} f(x)\right|}{\log \log n}=\beta\right\} .
$$

As indicated in Proposition 1.3 the size of the level sets are measured using the following refinement of the Hausdorff dimension.

Definition 1.4. - Let $E \subset \mathbb{R}^{d}$. We say that $E$ has precised Hausdorff dimension $(\alpha, \beta)$ if $\alpha$ is the Hausdorff dimension of $E$ and

- $\beta=0$ if $\mathscr{H}^{\phi_{\alpha, t}}(E)=0$ for every $t \in(0,1)$;

- $\beta=\sup \left\{t \in(0,1) ; \mathcal{H}^{\phi_{\alpha, t}}(E)>0\right\}$ otherwise. 
The precised Hausdorff dimension is a tool to classify sets that have the same Hausdorff dimension. The natural order for the precised dimension $(s, t)$ is the lexicographical order which will be denoted by $\prec$. With respect to this order, we can say that the greater is the set, the greater is the precised dimension. Moreover, if $(s, t) \prec\left(s^{\prime}, t^{\prime}\right)$ and $(s, t) \neq\left(s^{\prime}, t^{\prime}\right)$, then $\phi_{s^{\prime}, t^{\prime}} \ll \phi_{s, t}$. It follows that $\mathcal{H}^{\phi_{s^{\prime}, t^{\prime}}}(E)=0$ as soon as $\mathcal{H}^{\phi_{s, t}}(E)<\infty$.

Our main theorem on $\mathscr{C}(\mathbb{T})$ is the following:

THEOREM 1.5. - The following statements are true.

(1) For all functions $f \in \mathscr{C}(\mathbb{T})$, for any $\beta \in[0,1]$, the precised Hausdorff dimension of $F(\beta, f)$ is at most $(1,1-\beta)$.

(2) For quasi-all functions $f \in \mathscr{C}(\mathbb{T})$, for any $\beta \in[0,1]$, the precised Hausdorff dimension of $F(\beta, f)$ is equal to $(1,1-\beta)$. In particular $\operatorname{dim}_{\mathcal{H}}(F(\beta, f))=1$.

The paper is organized as follows. In the remaining part of this section, we introduce tools which will be needed during the rest of the paper. In Section 2, we prove Theorem 1.2 whereas in Section 3, we prove Theorem 1.5.

We conclude this introduction by mentioning that the companion problem of obtaining similar results for genericity in the sense of prevalence is considered in [2].

\subsection{A precised version of Fejér's theorem}

Working on Fourier series, we will need results on approximation by trigonometric polynomials. Let $k \in \mathbb{Z}$ and $e_{k}: t \mapsto e^{2 \pi i k t}$, so that, for any $g \in L^{1}(\mathbb{T})$ and any $n \in \mathbb{N}$,

$$
S_{n} g: t \mapsto \sum_{k=-n}^{n}\left\langle g, e_{k}\right\rangle e_{k}(t)
$$

Let $\sigma_{n} g$ be the $n$-th Fejér sum of $g$,

$$
\sigma_{n} g: t \mapsto \frac{1}{n} \sum_{k=0}^{n-1} S_{k} g(t)
$$

$\sigma_{n} g$ is obtained by taking the convolution of $g$ with the Fejér kernel

$$
F_{n}: t \mapsto \frac{1}{n}\left(\frac{\sin (n \pi t)}{\sin (\pi t)}\right)^{2} .
$$

If $g$ belongs to $\mathscr{C}(\mathbb{T}),\left(\sigma_{n} g\right)_{n \geq 1}$ converges uniformly to $g$. For our purpose, we need to estimate how quick the convergence is. This is the content of the next lemma (part (1) rectifies a mistake in the proof of Lemma 12 in [1] and requires to replace $\|\theta\|_{\infty} / 4$ in Aubry's version by $\left.\|\theta\|_{\infty} / 2\right)$.

Lemma 1.6. - Let $\theta$ be a Lipschitz function on $\mathbb{T}$, let $n \in \mathbb{N}$ and let $x \in \mathbb{T}$. Suppose that $\left\|\theta^{\prime}\right\|_{\infty} \leq n$ and that $\theta(x)=0$. Then the two following inequalities hold:

$$
\begin{aligned}
& \left|\sigma_{n} \theta(x)\right| \leq \frac{1}{4}+\frac{1}{2}\|\theta\|_{\infty} \text { for any } n \geq 8 \\
& \left|\sigma_{n} \theta(x)\right| \leq 4+\frac{1}{4}\|\theta\|_{\infty} \text { for any } n \geq 4 .
\end{aligned}
$$

$4^{\mathrm{e}}$ SÉRIE - TOME $45-2012-\mathrm{N}^{\circ} 6$ 
Proof. - We may assume that $x=0$. Hence, $\sigma_{n} \theta(0)=\int_{-1 / 2}^{1 / 2} \theta(y) F_{n}(y) d y$. Let us consider $\delta \in(0,2]$ and $n \geq 4$. On the one hand, for any $y \in[0,1 / 2)$,

$$
0 \leq F_{n}(y)=\frac{\sin ^{2}(n \pi y)}{n \sin ^{2}(\pi y)} \leq \frac{1}{n(2 y)^{2}}
$$

so that

$$
\left|\int_{\delta / n<|y| \leq 1 / 2} \theta(y) F_{n}(y) d y\right| \leq \frac{1}{2 n}\|\theta\|_{\infty} \int_{\delta / n}^{+\infty} \frac{d y}{y^{2}}=\frac{\|\theta\|_{\infty}}{2 \delta} .
$$

On the other hand,

$$
\left|\int_{-\delta / n}^{\delta / n} \theta(y) F_{n}(y) d y\right| \leq 2 \int_{0}^{\delta / n}\left(\frac{\sin (n \pi y)}{\sin (\pi y)}\right)^{2} y d y:=u_{n} .
$$

Using the convexity inequality $\sin \left(\frac{n}{n+1} \pi y\right) \geq \frac{n}{n+1} \sin (\pi y)$ and a change of variables, we see that $\left(u_{n}\right)$ is non-increasing. To prove (1), we choose $\delta=1$ and we observe that $u_{8}=0.2496 \ldots \leq \frac{1}{4}$. To prove (2), we choose $\delta=2$ and we observe that, since the maximum of $F_{n}$ is $F_{n}(0)=n$,

$$
\left|u_{n}\right| \leq 2 n^{2} \int_{0}^{2 / n} y d y=4
$$

\subsection{The mass transference principle}

The second main tool that we need in this paper is a method to produce sets with large Hausdorff dimension (Theorem 1.2) or with large precised Hausdorff dimension (Theorem 1.5). An efficient way to do this is to consider ubiquitous systems like this was done in $[4,6]$. This was later refined in [3] to obtain a general mass transference principle, which we recall in the form that we need.

THEOREM 1.7 (The mass transference principle). - Let $\left(x_{n}\right)_{n \geq 0}$ be a sequence of points in $[0,1]^{d}$ and let $\left(r_{n}\right)_{n \geq 0}$ be a sequence of positive real numbers decreasing to 0 . Let also $\phi: \mathbb{R}_{+} \rightarrow \mathbb{R}_{+}$be a dimension function satisfying $\phi(s) \gg s^{d}$ when $s$ goes to 0 and $s^{-d} \phi$ is monotonic. Define

$$
\begin{aligned}
E & =\limsup _{n} B\left(x_{n}, r_{n}\right) \\
E^{\phi} & =\limsup _{n} B\left(x_{n}, \phi^{-1}\left(r_{n}^{d}\right)\right)
\end{aligned}
$$

and suppose that almost every point of $[0,1]^{d}$ (in the sense of the Lebesgue's measure) lies in $E$. Then, $\mathcal{H}^{\phi}\left(E^{\phi}\right)=+\infty$.

We shall use it in the following situation.

COROLlary 1.8. - Let $\left(q_{n}\right)$ be a sequence of integers and, for each $n \in \mathbb{N}$, each $k \leq q_{n}$, let $B_{k, n}=B\left(x_{k, n}, r_{n}\right)$ be a ball with center $x_{k, n} \in[0,1]^{d}$ and with radius $r_{k, n}$ such that $\lim _{n \rightarrow+\infty} \max _{k}\left(r_{k, n}\right)=0$. Let also $\phi: \mathbb{R}_{+} \rightarrow \mathbb{R}_{+}$be a dimension function satisfying $\phi(s) \gg s^{d}$ when $s$ goes to 0 and $s^{-d} \phi$ is monotonic. Define

$$
\begin{array}{rlrl}
B_{n} & =\bigcup_{k=1}^{q_{n}} B_{k, n} & E & =\lim \sup _{n} B_{n} \\
B_{n}^{\phi} & =\bigcup_{k=1}^{q_{n}} B\left(x_{k, n}, \phi^{-1}\left(r_{k, n}^{d}\right)\right) & E^{\phi} & =\lim \sup _{n} B_{n}^{\phi} .
\end{array}
$$


Suppose that almost every point of $[0,1]^{d}$ (in the sense of the Lebesgue's measure) lies in $E$. Then, $\mathcal{H}^{\phi}\left(E^{\phi}\right)=+\infty$.

Proof. - Reordering the sequences $\left(B_{k, n}\right)$ and $\left(B_{k, n}^{\phi}\right)$ as $\left(C_{j}\right)$ and $\left(C_{j}^{\phi}\right)$, we can observe that

$$
\begin{aligned}
& \limsup _{n} B_{n}=\limsup _{j} C_{j}=E \\
& \limsup _{n} B_{n}^{\phi}=\limsup _{j} C_{j}^{\phi}=E^{\phi} .
\end{aligned}
$$

Thus the corollary follows from a direct application of Theorem 1.7.

\section{Multifractal analysis of the divergence of the Fourier series of functions of $L^{p}(\mathbb{T})$}

In this section, we shall prove Theorem 1.2. Our method, which is inspired by [7], is divided into two parts. During the first one, we will construct a single function, which we call the saturating function, satisfying the conclusions of Theorem 1.2. During the second one, we will show how to derive a residual set from this single function.

\subsection{The saturating function}

Our intention is to construct a function $g$ such that $\left|S_{n} g(x)\right|$ is big when $x$ is close to a dyadic number. The following definition gives a precise meaning.

Definition 2.1. - A real number $x$ is $\alpha$-approximable by dyadics, $\alpha \geq 1$, if there exist two sequences of integers $\left(k_{n}\right),\left(j_{n}\right)$ such that

$$
\left|x-\frac{k_{n}}{2^{j_{n}}}\right| \leq \frac{1}{2^{\alpha j_{n}}}
$$

and $\left(j_{n}\right)$ goes to infinity. The dyadic exponent of $x$ is the supremum of the set of real numbers $\alpha$ such that $x$ is $\alpha$-approximable by dyadics.

We denote by

$$
D_{\alpha}=\{x \in[0,1] ; x \text { is } \alpha \text {-approximable by dyadics }\} .
$$

It is easy to check that $\mathcal{H}^{\beta}\left(D_{\alpha}\right)=0$ for $\beta>1 / \alpha$ so that $\operatorname{dim}_{\mathcal{H}}\left(D_{\alpha}\right) \leq 1 / \alpha$. On the other hand, it is well-known that $\operatorname{dim}_{\mathcal{H}}\left(D_{\alpha}\right) \geq \frac{1}{\alpha}$.

Let us nevertheless show how this follows from Corollary 1.8. Indeed, $D_{\alpha}$ can be described as a limsup set:

where the $I_{k, j}$ are the dyadic intervals

$$
D_{\alpha}=\limsup _{j \rightarrow+\infty} \bigcup_{k=0}^{2^{j}-1} I_{k, j}^{\alpha}
$$

$$
I_{k, j}=\left[\frac{k}{2^{j}}-\frac{1}{2^{j}}, \frac{k}{2^{j}}+\frac{1}{2^{j}}\right]
$$

and

$$
I_{k, j}^{\alpha}=\left[\frac{k}{2^{j}}-\frac{1}{2^{\alpha j}}, \frac{k}{2^{j}}+\frac{1}{2^{\alpha j}}\right] .
$$

$4^{\text {e }}$ SÉRIE - TOME $45-2012-\mathrm{N}^{\circ} 6$ 
Since $\bigcup_{k=0}^{2^{j}-1} I_{k, j} \supset[0,1]$, Corollary 1.8 implies that $\mathcal{H}^{1 / \alpha}\left(D_{\alpha}\right)=+\infty$.

We are going to define $g \in L^{p}(\mathbb{T})$ such that the divergence index of $g$ at $x$ depends on the dyadic exponent of $x$. The greater the dyadic exponent will be, the greater the divergence index of $g$ at $x$ will be. To do this, we will classify the dyadic intervals following their center. Namely, each $k / 2^{j}$ can be uniquely written $K / 2^{J}$ with $K \notin 2 \mathbb{Z}$ and $1 \leq J \leq j$ (such a center comes into play from the $J$-th generation). Let $\mathcal{J}_{J}=\left\{K / 2^{J} ; K \notin 2 \mathbb{Z}, 0 \leq K \leq 2^{J}-1\right\}$ and

$$
\mathbf{I}_{J, j}=\bigcup_{\frac{k}{2^{j}} \in \mathcal{J}_{J}} I_{k, j} \quad \mathbf{I}_{J, j}^{\prime}=\bigcup_{\frac{k}{2^{j}} \in \mathcal{J}_{J}} 2 I_{k, j} .
$$

Here and elsewhere, when $I$ is an interval and $c$ is a positive real number, $c I$ means the interval with the same center as $I$ and with length $c|I|$. Observe that, when $1 \leq J<j$, the intervals $2 I_{k, j}, \frac{k}{2^{j}} \in J_{J}$ do not overlap and the set $\mathbf{I}_{J, j}^{\prime}$ has measure $2^{J-1} 2^{2-j}$. Observe also that when $J$ is small with respect to $j$, the real numbers $x$ in $\mathbf{I}_{J, j}$ are well-approximated by dyadics $K / 2^{J}$, since $\left|x-K / 2^{J}\right| \leq 1 / 2^{j}$.

We first define a trigonometric polynomial with $L^{p}$-norm 1 which is almost constant on each $\mathscr{J}_{J, j}$ and which is big on $\mathcal{J}_{J, j}$ when $J$ is small.

Lemma 2.2. - Let $j \geq 1$. There exists a trigonometric polynomial $g_{j} \in L^{p}(\mathbb{T})$ with Fourier spectrum contained in $\left[0, j 2^{j+1}\right)$ such that

- $\left\|g_{j}\right\|_{p} \leq 1$

- For any $1 \leq J \leq j$ and any $x \in \mathbf{I}_{J, j}$, we can find two integers $n_{1}$ and $n_{2}$ satisfying $0 \leq n_{1}<n_{2}<j 2^{j+1}$ and such that

$$
\left|S_{n_{2}} g_{j}(x)-S_{n_{1}} g_{j}(x)\right| \geq \frac{1}{4 j} 2^{-(J-j+1) / p} .
$$

Proof. - We set for any $1 \leq J \leq j$ :

- $\chi_{J, j}$ a continuous piecewise linear function equal to 1 on $\mathbf{I}_{J, j}$, equal to 0 outside $\mathbf{I}_{J, j}^{\prime}$, and satisfying $0 \leq \chi_{J, j} \leq 1$ and $\left\|\chi_{J, j}^{\prime}\right\|_{\infty} \leq 2^{j}$;

- $c_{J, j}=\frac{1}{j} 2^{-(J-j+1) / p}\left(c_{J, j}\right.$ is big when $J$ is small);

- $g_{J, j}=e_{(2 J-1) 2^{j}} \sigma_{2^{j}} \chi_{J, j}$.

It is straightforward to observe that the Fourier spectrum of $g_{J, j}$ is contained in $\left[n_{J, j}, m_{J, j}\right]$ with

$$
\left\{\begin{aligned}
n_{J, j} & =(2 J-1) 2^{j}-\left(2^{j}-1\right) \\
m_{J, j} & =(2 J-1) 2^{j}+\left(2^{j}-1\right) .
\end{aligned}\right.
$$

Thus, the Fourier spectra of the $g_{J, j}, 1 \leq J \leq j$ are disjoint. Moreover, $\left\|g_{j, j}\right\|_{p}=1$ and for $1 \leq J<j,\left\|g_{J, j}\right\|_{p} \leq\left\|\chi_{J, j}\right\|_{p} \leq 2^{(J-j+1) / p}$.

We finally set

$$
g_{j}=\sum_{J=1}^{j} c_{J, j} g_{J, j}
$$


and we claim that $g_{j}$ is the trigonometric polynomial we are looking for. First of all, the Fourier spectrum of $g_{j}$ is included in $\left[n_{1, j}, m_{j, j}\right]$ which is contained in $\left[0, j 2^{j+1}\right)$. Moreover, the $L^{p}$ norm of $g_{j}$ is

$$
\left\|g_{j}\right\|_{p} \leq \sum_{J=1}^{j} \frac{1}{j} 2^{-(J-j+1) / p}\left\|g_{J, j}\right\|_{p} \leq 1 .
$$

Pick now any $x \in \mathbf{I}_{J, j}, 1 \leq J \leq j$ so that

$$
\begin{aligned}
\left|S_{m_{J, j}} g_{j}(x)-S_{n_{J, j}-1} g_{j}(x)\right| & =\left|c_{J, j} g_{J, j}(x)\right| \\
& =\frac{1}{j} 2^{-(J-j+1) / p}\left|\sigma_{2^{j}} \chi_{J, j}(x)\right| .
\end{aligned}
$$

Observing that $\chi_{J, j}(x)=1$ and applying the first point of Lemma 1.6 to $1-\chi_{J, j}$, we find

$$
\left|\sigma_{2^{j}} \chi_{J, j}(x)\right| \geq 1-\left|\sigma_{2^{j}}\left(1-\chi_{J, j}(x)\right)\right| \geq \frac{1}{4} .
$$

Thus,

$$
\left|S_{m_{J, j}} g_{j}(x)-S_{n_{J, j}-1} g_{j}(x)\right| \geq \frac{1}{4 j} 2^{-(J-j+1) / p}
$$

and the conclusion follows with $n_{2}=m_{J, j}$ and $n_{1}=n_{J, j}-1$.

We are now ready to construct the saturating function. It is defined by

$$
g=\sum_{j \geq 1} \frac{1}{j^{2}} e_{j 2^{j+1}} g_{j}
$$

Observe in particular that the functions $e_{j 2^{j+1}} g_{j}$ have disjoint Fourier spectra (the Fourier spectrum of $e_{j 2^{j+1}} g_{j}$ is contained in $\left[j 2^{j+1} ; j 2^{j+2}\right)$ ) and that $g$ belongs to $L^{p}(\mathbb{T})$.

We then show that for any $x \in D_{\alpha}, \alpha>1$,

$$
\limsup _{n \rightarrow+\infty} \frac{\log \left|S_{n} g(x)\right|}{\log n} \geq \frac{1}{p}\left(1-\frac{1}{\alpha}\right) \text {. }
$$

Indeed, let $x \in D_{\alpha}$ and let $\varepsilon>0$ with $\alpha-\varepsilon>1$. We can find integers $K$ and $J$ with $J$ as large as we want and $K \notin 2 \mathbb{Z}$ such that

$$
\left|x-\frac{K}{2^{J}}\right| \leq \frac{1}{2^{(\alpha-\varepsilon / 2) J}} .
$$

We set $j=[(\alpha-\varepsilon / 2) J]$ the integer part of $(\alpha-\varepsilon / 2) J$ and $k$ such that $k / 2^{j}=K / 2^{J}$. Hence,

$$
\left|x-\frac{k}{2^{j}}\right| \leq \frac{1}{2^{(\alpha-\varepsilon / 2) J}} \leq \frac{1}{2^{j}} .
$$

Using Lemma 2.2, we can find two integers $n_{1}$ and $n_{2}$ satisfying $j 2^{j+1} \leq n_{1}<n_{2}<j 2^{j+2}$ and such that

$$
\begin{aligned}
\left|S_{n_{2}} g(x)-S_{n_{1}} g(x)\right| & =\frac{1}{j^{2}}\left|S_{n_{2}}\left(e_{j 2^{j+1}} g_{j}\right)(x)-S_{n_{1}}\left(e_{j 2^{j+1}} g_{j}\right)(x)\right| \\
& \geq \frac{1}{4 j^{3}} 2^{-(J-j+1) / p} \\
& \geq \frac{1}{4 j^{3}} 2^{\frac{1}{p}\left(j-\frac{j+1}{\alpha-\varepsilon / 2}-1\right)} \\
& \geq C 2^{\frac{1}{p}\left(1-\frac{1}{\alpha-\varepsilon}\right) j}
\end{aligned}
$$


It follows that we can find $n \in\left\{n_{1}, n_{2}\right\}$ such that $\left|S_{n} g(x)\right| \geq \frac{C}{2} 2^{\frac{1}{p}\left(1-\frac{1}{\alpha-\varepsilon}\right) j}$. Combining the estimates on $n$ and on $\left|S_{n} g(x)\right|$, and since $J$ (hence $j$, hence $n$ ) can be taken as large as we want, we get that

$$
\limsup _{n \rightarrow+\infty} \frac{\log \left|S_{n} g(x)\right|}{\log n} \geq \frac{1}{p}\left(1-\frac{1}{\alpha-\varepsilon}\right) .
$$

Since $\varepsilon>0$ is arbitrary, we obtain in fact that

$$
\text { for any } x \in D_{\alpha}, \quad \limsup _{n \rightarrow+\infty} \frac{\log \left|S_{n} g(x)\right|}{\log n} \geq \frac{1}{p}\left(1-\frac{1}{\alpha}\right) \text {. }
$$

At this point, it would be nice to get a lower bound for $\lim \sup _{n \rightarrow+\infty} \frac{\log \left|S_{n} g(x)\right|}{\log n}$ for any $x$ with dyadic exponent equal to $\alpha$. Unfortunately, this does not seem easy and we will rather conclude by using an argument lying on Hausdorff measures. Indeed, define

$$
\begin{aligned}
& D_{\alpha}^{1}=\left\{x \in D^{\alpha} ; \limsup _{n \rightarrow+\infty} \frac{\log \left|S_{n} g(x)\right|}{\log n}=\frac{1}{p}\left(1-\frac{1}{\alpha}\right)\right\} \\
& D_{\alpha}^{2}=\left\{x \in D^{\alpha} ; \limsup _{n \rightarrow+\infty} \frac{\log \left|S_{n} g(x)\right|}{\log n}>\frac{1}{p}\left(1-\frac{1}{\alpha}\right)\right\} .
\end{aligned}
$$

We have already observed that $\mathcal{H}^{1 / \alpha}\left(D_{\alpha}^{1} \cup D_{\alpha}^{2}\right)=\mathcal{H}^{1 / \alpha}\left(D_{\alpha}\right)=+\infty$. It suffices to prove that $\mathcal{H}^{1 / \alpha}\left(D_{\alpha}^{2}\right)=0$. Let $\left(\beta_{n}\right)$ be a sequence of real numbers such that $\beta_{n}>\frac{1}{p}\left(1-\frac{1}{\alpha}\right)$ and $\lim _{n \rightarrow+\infty} \beta_{n}=\frac{1}{p}\left(1-\frac{1}{\alpha}\right)$.

Let us observe that

$$
D_{\alpha}^{2} \subset \bigcup_{n \geq 0} \mathcal{E}\left(\beta_{n}, g\right) .
$$

Moreover, Theorem 1.1 implies that $\mathcal{H}^{1 / \alpha}\left(\mathcal{E}\left(\beta_{n}, g\right)\right)=0$ for all $n$. Hence, $\mathcal{H}^{1 / \alpha}\left(D_{\alpha}^{2}\right)=0$ and $\mathcal{H}^{1 / \alpha}\left(D_{\alpha}^{1}\right)=+\infty$, which proves that

$$
\operatorname{dim}_{\mathscr{H}}\left(E\left(\frac{1}{p}\left(1-\frac{1}{\alpha}\right), g\right)\right) \geq \frac{1}{\alpha}
$$

By Theorem 1.1 again, this inequality is necessarily an equality. Finally, $g$ satisfies the conclusions of Theorem 1.2, setting $1-\beta p=1 / \alpha$.

REMARK 2.3. - If $\alpha=1$, then $\beta=0$ and the conclusion is a consequence of Carleson's Theorem.

\subsection{The residual set}

To build the dense $G_{\delta}$-set, the idea is that any function whose Fourier coefficients are sufficiently close to those of the saturating function $g$ on infinitely many intervals $\left[j 2^{j+1} ; j 2^{j+2}\right.$ ) will satisfy the conclusions of Theorem 1.2. Precisely, let $\left(f_{j}\right)_{j \geq 1}$ be a dense sequence of polynomials in $L^{p}(\mathbb{T})$ with Fourier spectrum contained in $[-j, j]$. We define a sequence $\left(h_{j}\right)_{j \geq 1}$ as follows:

$$
h_{j}=f_{j}+\frac{1}{j} e_{j 2^{j+1}} g_{j}
$$

so that $\left\|h_{j}-f_{j}\right\|_{p}$ goes to 0 and $\left(h_{j}\right)_{j \geq 1}$ remains dense in $L^{p}(\mathbb{T})$. Observe also that the Fourier spectra of $f_{j}$ and $h_{j}-f_{j}$ do not overlap. Finally, let $\left(r_{j}\right)_{j \geq 1}$ be a sequence of positive integers 
so small that, for any $f \in L^{p}(\mathbb{T})$ with $\|f\|_{L^{p}} \leq r_{j},\left\|S_{n} f\right\|_{\infty} \leq 1$ for any $n \leq j 2^{j+2}$. The dense $G_{\delta}$ set we will consider is

$$
A=\bigcap_{l \in \mathbb{N} j \geq l} B\left(h_{j}, r_{j}\right)
$$

Let $f$ belong to $A$ and let $\left(j_{l}\right)_{l \geq 1}$ be an increasing sequence of integers such that $f$ belongs to $B\left(h_{j_{l}}, r_{j_{l}}\right)$ for any $l$. Then, for any $\alpha>1$, we define $J_{l}=\left[j_{l} / \alpha\right]+1$ (which is smaller than $j_{l}$ if $l$ is large enough) and

$$
E=\limsup _{l \rightarrow+\infty} \mathbf{I}_{J_{l}, j_{l}} .
$$

For any $x \in E$ one can find $j=j_{l}$ as large as we want, the corresponding $J=J_{l}$ and $1 \leq k \leq 2^{j}-1$ such that $x$ belongs to $I_{k, j}$ with $k / 2^{j} \in \mathcal{J}_{J}$.

Observe that $f=f_{j}+\frac{1}{j} e_{j 2^{j+1}} g_{j}+\left(f-h_{j}\right)$. By Lemma 2.2, we can find two integers $n_{1}$ and $n_{2}$ satisfying $j 2^{j+1} \leq n_{1}<n_{2}<j 2^{j+2}$ and such that

$$
\left|S_{n_{2}}\left(e_{j 2^{j+1}} g_{j}\right)(x)-S_{n_{1}}\left(e_{j 2^{j+1}} g_{j}\right)(x)\right| \geq \frac{1}{4 j} 2^{-(J-j+1) / p} .
$$

Using the definition of the $r_{j}$, we obtain

$$
\begin{aligned}
\left|S_{n_{2}} f(x)-S_{n_{1}} f(x)\right| & \geq \frac{1}{4 j^{2}} 2^{-(J-j+1) / p}-\left|S_{n_{2}}\left(f-h_{j}\right)(x)\right|-\left|S_{n_{1}}\left(f-h_{j}\right)(x)\right| \\
& \geq \frac{1}{4 j^{2}} 2^{-(J-j+1) / p}-2
\end{aligned}
$$

so that

$$
\left|S_{n_{2}} f(x)\right| \geq \frac{C}{j^{2}} 2^{-(J-j+1) / p} \quad \text { or } \quad\left|S_{n_{1}} f(x)\right| \geq \frac{C}{j^{2}} 2^{-(J-j+1) / p} .
$$

Observing that

$$
\left\{\begin{aligned}
\max \left(\log n_{2}, \log n_{1}\right) & =j \log 2+O(\log j) \\
\log \left(j^{-2} 2^{-(J-j+1) / p}\right) & =\frac{1}{p}\left(1-\frac{1}{\alpha}\right) j \log 2+O(\log j)
\end{aligned}\right.
$$

we find in particular that, for any $x \in E$,

$$
\limsup _{n \rightarrow+\infty} \frac{\log \left|S_{n} f(x)\right|}{\log n} \geq \frac{1}{p}\left(1-\frac{1}{\alpha}\right) .
$$

On the other hand, let us write

$$
\mathbf{I}_{J_{l}, j_{l}}=\bigcup_{1 \leq K<2^{J_{l}}, K \notin 2 \mathbb{Z}}\left[\frac{K}{2^{J_{l}}}-\frac{1}{2^{j_{l}}}, \frac{K}{2^{J_{l}}}+\frac{1}{2^{j_{l}}}\right]
$$

and remark that for any $l$, since $J_{l} \geq j_{l} / \alpha$,

$$
\bigcup_{1 \leq K<2^{J_{l}}, K \notin 2 \mathbb{Z}}\left[\frac{K}{2^{J_{l}}}-\frac{1}{2^{j_{l} / \alpha}}, \frac{K}{2^{J_{l}}}+\frac{1}{2^{j_{l} / \alpha}}\right] \supset[0,1] .
$$

Hence, we can apply Corollary 1.8 to get $\mathcal{H}^{1 / \alpha}(E)=+\infty$. We now conclude exactly as in Section 2.1 to get $\mathcal{H}^{1 / \alpha}\left(E^{1}\right)=+\infty$, with

$$
E^{1}=\left\{x \in E ; \limsup _{n \rightarrow+\infty} \frac{\log \left|S_{n} f(x)\right|}{\log n}=\frac{1}{p}\left(1-\frac{1}{\alpha}\right)\right\}
$$


Finally,

$$
\operatorname{dim}_{\mathcal{H}}\left(E\left(\frac{1}{p}\left(1-\frac{1}{\alpha}\right), f\right)\right) \geq \frac{1}{\alpha}
$$

and $f$ satisfies the conclusions of Theorem 1.2, setting $1-\beta p=1 / \alpha$.

ReMARK 2.4. - During the construction, we did not use that the Fourier spectra of the functions $e_{j 2^{j+1}} g_{j}$ are disjoint, because we considered each one separately. We could also define $h_{j}$ by $h_{j}=f_{j}+\frac{1}{j} e_{j+1} g_{j}$.

REMARK 2.5. - The above construction can be carried on $L^{1}(\mathbb{T})$. Namely, for quasi-all $f \in L^{1}(\mathbb{T})$, we obtain for any $\beta \in[0,1]$,

$$
\operatorname{dim}_{\mathcal{H}}(E(\beta, f)) \geq 1-\beta .
$$

However, the Hunt-Carleson's maximal inequality dramatically breaks down in $L^{1}(\mathbb{T})$ and Aubry's method is not relevant in this context. In a recent paper, we tackled this difficulty and obtained an analogue of Theorem 1.1 in the $L^{1}$ context, thus extending Theorem 1.2 when $p=1$ (see [2]).

\section{Multifractal analysis of the divergence of the Fourier series of functions of $\mathscr{C}(\mathbb{T})$}

We turn to the proof of Theorem 1.5. We follow a strategy close to that of Section 2. First of all, we will give an upper bound for the precised Hausdorff dimension of the sets $\mathcal{F}(\beta, f)$ (hence, of the sets $F(\beta, f)$ ) for any $f \in \mathscr{C}(\mathbb{T})$ and any $\beta \in(0,1)$. Second, we will build polynomials with small $L^{\infty}$-norms and such that their Fourier series have big partial sums on big intervals. These polynomials will be the blocks of our final construction. Working on $\mathscr{C}(\mathbb{T})$ adds several difficulties which will be explained when we will encounter them.

\subsection{The sets $\mathcal{F}(\beta, f)$ cannot be too big}

We begin with the following lemma which completes Proposition 1.3 (recall that $\left.\phi_{s, t}(x)=x^{s} \exp \left((\log 1 / x)^{1-t}\right)\right)$.

Lemma 3.1. - Let $\beta \in(0,1)$ and $f \in \mathscr{C}(\mathbb{T})$. Then, for any $\gamma>1-\beta$,

$$
\mathcal{H}^{\phi_{1, \gamma}}(\mathcal{F}(\beta, f))=0 \text {. }
$$

In particular, the precised Hausdorff dimension of $\mathcal{F}(\beta, f)$ and $F(\beta, f)$ cannot exceed $(1,1-\beta)$.

Proof. - A key point in Aubry's proof of Theorem 1.1 is the Carleson-Hunt theorem which asserts that, for any $g \in L^{p}(\mathbb{T}), 1<p<+\infty$,

$$
\left\|S^{*} g\right\|_{p} \leq C_{p}\|g\|_{p} \quad \text { where } \quad S^{*} g(x)=\sup _{n \geq 0}\left|S_{n} g(x)\right| .
$$

On $\mathscr{C}(\mathbb{T})$, a weak inequality (also due to Hunt) remains valid (see [13, Theorem 12.5]): there are two absolute constants $A, B>0$ such that, for every $f \in \mathscr{C}(\mathbb{T})$ and every $y>0$,

$$
\lambda\left(\left\{x \in \mathbb{T} ; S^{*} f(x)>y\right\}\right) \leq A e^{-B y /\|f\|_{\infty}} .
$$

Here, $\lambda$ denotes the Lebesgue measure on $\mathbb{T}$. 
So, let $\beta \in(0,1)$ and $f \in \mathscr{C}(\mathbb{T})$. We may assume $\|f\|_{\infty} \leq 1$. For any $M>0$, we introduce

$$
\mathcal{F}(\beta, f, M)=\left\{x \in \mathbb{T} ; \limsup _{n \rightarrow+\infty}(\log n)^{-\beta}\left|S_{n} f(x)\right|>M\right\} .
$$

Since $\mathcal{F}(\beta, f)=\bigcup_{M>0} \mathcal{F}(\beta, f, M)$, we just need to prove that $\mathcal{H}^{\phi_{1, \gamma}}(\mathcal{F}(\beta, f, M))=0$ for every $M>0$. From now on, we fix some $M>0$. We pick any $x \in \mathcal{F}(\beta, f, M)$ and $n_{x}$ large enough such that

$$
\left|S_{n_{x}} f(x)\right| \geq M\left(\log n_{x}\right)^{\beta} .
$$

Such an inequality remains true in an interval around $x$ whose size is not so small. Precisely, because $n_{x}$ can be assumed to be large and since the $L^{1}$-norm of the Dirichlet kernel $D_{n}$ behaves like $\frac{4}{\pi^{2}} \log n$, we may assume that $\left\|S_{n_{x}} f\right\|_{\infty} \leq\left(\log n_{x}\right)\|f\|_{\infty} \leq \log n_{x}$. By Bernstein's inequality, $\left\|\left(S_{n_{x}} f\right)^{\prime}\right\|_{\infty} \leq n_{x} \log n_{x}$. Let

$$
I_{x}=\left[x-\frac{M}{2 n_{x}\left(\log n_{x}\right)^{1-\beta}}, x+\frac{M}{2 n_{x}\left(\log n_{x}\right)^{1-\beta}}\right] .
$$

For any $y \in I_{x}$, we get

$$
\left|S_{n_{x}} f(y)\right| \geq \frac{M}{2}\left(\log n_{x}\right)^{\beta} .
$$

$\left(I_{x}\right)_{x \in \mathcal{F}(\beta, f, M)}$ is a covering of $\mathcal{F}(\beta, f, M)$. We can extract a Vitali's covering, namely a countable family of disjoint intervals $I_{i}, i \in \mathbb{N}$, of length $\frac{M}{n_{i}\left(\log n_{i}\right)^{1-\beta}}$ such that $\mathcal{F}(\beta, f, M) \subset \bigcup_{i} 5 I_{i}$. Let us finally set, for any $q \geq 1, \mathcal{U}_{q}=\left\{i ; 2^{q+1} \geq \frac{M\left(\log n_{i}\right)^{\beta}}{2}>2^{q}\right\}$. Without loss of generality, we may assume the $n_{i}$ so large that $\bigcup_{q} \mathcal{U}_{q}=\mathbb{N}$. By applying Hunt's theorem,

$$
\lambda\left(\left\{x ; S^{*} f(x)>2^{q}\right\}\right) \leq A e^{-B 2^{q}} .
$$

Now, by (3), the set $\left\{x ; S^{*} f(x)>2^{q}\right\}$ contains the disjoint intervals $I_{i}$, for $i \in \mathcal{U}_{q}$. Thus,

$$
\sum_{i \in U_{q}}\left|I_{i}\right| \leq A e^{-B 2^{q}}
$$

Moreover, for any $i \in \mathcal{U}_{q}$, it is not hard to check that

$$
\left|I_{i}\right| \geq C e^{-D 2^{q / \beta}}
$$

for some positive constants $C, D$ which do not depend on $q$. Picking any $\alpha$ such that $1-\beta<\alpha<\gamma$, we get

$$
\begin{aligned}
\sum_{i \in \mathcal{U}_{q}} \phi_{1, \alpha}\left(5\left|I_{i}\right|\right) & =\sum_{i \in \mathcal{U}_{q}} 5\left|I_{i}\right| \exp \left(\left(\log \left(1 / 5\left|I_{i}\right|\right)\right)^{1-\alpha}\right) \\
& \leq 5\left(\sum_{i \in \mathcal{U}_{q}}\left|I_{i}\right|\right) \exp \left(\left(D 2^{q / \beta}-\log 5 C\right)^{1-\alpha}\right) \\
& \leq 5 A e^{-B 2^{q}+D^{\prime} 2^{q(1-\alpha) / \beta}}
\end{aligned}
$$

Since $1-\alpha<\beta$, this shows that there exists $C_{0}<+\infty$ such that

$$
\sum_{i \in \mathbb{N}} \phi_{1, \alpha}\left(5\left|I_{i}\right|\right)=\sum_{q \in \mathbb{N}} \sum_{i \in \mathcal{U}_{q}} \phi_{1, \alpha}\left(5\left|I_{i}\right|\right) \leq C_{0} .
$$

$4^{\text {e }}$ SÉRIE - TOME $45-2012-$ No $^{\circ} 6$ 
Remember that $\bigcup_{i} 5 I_{i}$ is a covering of $\mathcal{F}(\beta, f, M)$ and that the $I_{i}$ can be chosen as small as we want. We can then conclude that $\mathcal{H}^{\phi_{1, \alpha}}(\mathcal{F}(\beta, f, M)) \leq C_{0}$. In particular, $\mathcal{H}^{\phi_{1, \gamma}}(\mathcal{F}(\beta, f, M))=0$, since $\phi_{1, \alpha} \gg \phi_{1, \gamma}$.

REMARK 3.2. - The functions $\phi_{1, \gamma}$, for $\gamma>1-\beta$, are not optimal in the statement of the previous lemma. We can replace them by any function $\phi(x)=x \exp \left((\log 1 / x)^{\beta} \varepsilon(x)\right)$ with $\varepsilon(x)$ going to 0 as $x$ goes to 0 .

\subsection{The basic construction}

When we try to build explicitly a continuous function whose Fourier series diverges at some point, say 0 , a natural way is to consider polynomials $P$ with small $L^{\infty}$ norm, and satisfying nevertheless that $\left|S_{n} P(0)\right|$ is big for some large value of $n$. The easiest examples are

$$
P_{N}(x)=e_{N}(x) \sum_{j=1}^{N} \frac{\sin (2 \pi j x)}{j},
$$

since the sequence $\left(\left\|P_{N}\right\|_{\infty}\right)_{N \geq 1}$ is bounded whereas $\left|S_{N}(P)(0)\right| \sim \frac{1}{2} \log N$. Considering series of the form $\sum_{j} \omega_{j} P_{N_{j}}$ and a sequence $\left(\varepsilon_{n}\right)$ decreasing to 0 , it is possible to obtain a continuous function $f$ such that $\lim \sup _{n \rightarrow+\infty} S_{n} f(0) /\left(\varepsilon_{n} \log n\right)=+\infty$. This is optimal since $S_{n} f(0)=o(\log n)$ for any $f \in \mathscr{C}(\mathbb{T})$ (see for example [14]).

In our context, we need to find a polynomial $P$ which satisfies a similar property not only at one point, but on a set which is rather big since at the end we want to construct sets of divergence with Hausdorff dimension 1 . This does not seem to be the case for $P_{N}$, the reason being that $\left|\left(S_{N} P\right)^{\prime}(0)\right|$ behaves like $N$, which is much bigger than $S_{N} P(0)$.

To tackle this problem, we start from a construction of Kahane and Katznelson in [8] (see also [10]) which they use to prove that every subset of $\mathbb{T}$ of Lebesgue measure 0 is a set of divergence for $\mathscr{C}(\mathbb{T})$. Since we want to control both the size of the sets $E$ and the index $n$ such that $S_{n} P(x)$ becomes larger than some given real number for any $x \in E$, the forthcoming lemma needs very careful estimations.

Lemma 3.3. - Let $\beta \in(0,1), \delta \in(0,1)$ and $K \geq 2$. Then there exist an integer $k \geq K$, an integer $n$ as large as we want and a trigonometric polynomial $P$ with Fourier spectrum contained in $[0,2 n-1]$ such that

$-|P(x)| \leq 1$ for any $x \in \mathbb{T}$;

- $\log \left|S_{n} P(x)\right| \geq(1-\delta) \beta \log \log n$ for any $x \in I_{k}^{\beta}$,

where $I_{k}^{\beta}=\bigcup_{j=0}^{k-1}\left[\frac{j}{k}-\frac{1}{2 k \exp \left((\log k)^{\beta}\right)}, \frac{j}{k}+\frac{1}{2 k \exp \left((\log k)^{\beta}\right)}\right]$.

Proof. - Let us first describe the idea of the proof. We shall construct a trigonometric polynomial $Q$ with Fourier spectrum in $[1, n-1]$ and with the following properties: $|\Im m Q|$ is small and $|Q|$ is large on a set $E$. We then set $P=e_{n} \times \Im m Q$, so that $\|P\|_{\infty}$ is small. On the other hand, writing $Q=\sum_{k=1}^{n-1} a_{k} e_{k}, 2 i \Im m Q=-\sum_{k=1}^{n-1} \overline{a_{k}} e_{-k}+\sum_{k=1}^{n-1} a_{k} e_{k}$, so that

$$
\left|S_{n}(P)\right|=\frac{1}{2}\left|\sum_{k=1}^{n-1} \overline{a_{k}} e_{n-k}\right|=\frac{1}{2}\left|\sum_{k=1}^{n-1} a_{k} e_{k}\right|=\frac{1}{2}|Q|
$$


is large on $E$. The construction of $Q$ will be done by taking $\log f$, the logarithm of a holomorphic function defined on a neighborhood of the closed unit disk $\overline{\mathbb{D}}$ (which allows to control the imaginary part of $\log f$ while its modulus can be large), and by taking a Fejér sum of $\log f$.

We now proceed with the details. The proof uses holomorphic functions and it is better to see $\mathbb{T}$ as the boundary of the unit disk $\mathbb{D}$. To avoid cumbersome notations, the letter $C$ will denote throughout the proof a positive and absolute constant, whose value may change from line to line. Let $k \geq K$ whose value will be fixed later. We set:

$$
\begin{aligned}
\varepsilon & =\frac{1}{k \exp \left((\log k)^{\beta}\right)} \\
z_{j} & =e^{\frac{2 \pi i j}{k}}, j=0, \ldots, k-1 \\
f(z) & =\frac{1}{k} \sum_{j=0}^{k-1} \frac{1+\varepsilon}{1+\varepsilon-\overline{z_{j}} z} .
\end{aligned}
$$

$f$ is holomorphic in a neighborhood of $\overline{\mathbb{D}}$. We claim that $f$ satisfies the following four properties.

(P1): $\forall z \in \overline{\mathbb{D}}, \quad \Re e f(z) \geq C \varepsilon$;

(P2): $\forall z \in I_{k}^{\beta}, \quad|f(z)| \geq \Re e f(z) \geq C \exp \left((\log k)^{\beta}\right)$;

(P3): $\forall z \in \mathbb{T}, \quad|f(z)| \leq C \exp \left((\log k)^{\beta}\right)$;

(P4): $\forall z \in \mathbb{T}, \quad\left|\frac{f^{\prime}(z)}{f(z)}\right| \leq \frac{C}{\varepsilon^{3}}$.

Indeed, for any $z \in \overline{\mathbb{D}}$ and any $j \in\{0, \ldots, k-1\}$,

$$
\Re e\left(\frac{1+\varepsilon}{1+\varepsilon-\overline{z_{j}} z}\right)=\frac{1+\varepsilon}{\left|1+\varepsilon-\overline{z_{j}} z\right|^{2}} \Re e\left(1+\varepsilon-z_{j} \bar{z}\right) \geq \frac{1+\varepsilon}{(2+\varepsilon)^{2}} \times \varepsilon \geq C \varepsilon,
$$

which proves (P1). To prove (P2), we may assume that $z=e^{2 \pi i \theta}$ with $\theta \in\left[\frac{-\varepsilon}{2} ; \frac{\varepsilon}{2}\right]$. Then

$$
\Re e\left(\frac{1+\varepsilon}{1+\varepsilon-\overline{z_{0}} z}\right)=\frac{1+\varepsilon}{|1+\varepsilon-z|^{2}} \Re e(1+\varepsilon-\bar{z}) \geq \frac{C}{\varepsilon} .
$$

If we combine this with (4), we get

$$
\Re e f(z) \geq \frac{C}{k \varepsilon}+\frac{k-1}{k} C \varepsilon \geq \frac{C}{k \varepsilon}=C \exp \left((\log k)^{\beta}\right) .
$$

which gives (P2).

Conversely, we want to control $\sup _{z \in \mathbb{T}}|f(z)|$. Pick any $z=e^{2 \pi i \theta} \in \mathbb{T}$. By symmetry, we may and shall assume that $|\theta| \leq \frac{1}{2 k}$. Then we get

$$
\left|\frac{1+\varepsilon}{1+\varepsilon-\overline{z_{0}} z}\right| \leq \frac{C}{\varepsilon}
$$

$4^{\text {e }}$ SÉRIE - TOME $45-2012-\mathrm{N}^{\circ} 6$ 
Now, for any $j \in\{1, \ldots, k / 4\}$, we can write

$$
\begin{aligned}
\left|1+\varepsilon-\overline{z_{j}} z\right| & \geq\left|\Im m\left(\overline{z_{j}} z\right)\right| \\
& \geq \sin \left(\frac{2 \pi j}{k}-2 \pi \theta\right) \\
& \geq \frac{2}{\pi} \times 2 \pi\left(\frac{j}{k}-\theta\right) \\
& \geq \frac{4}{k}\left(j-\frac{1}{2}\right) .
\end{aligned}
$$

Taking the sum,

$$
\left|\sum_{j=1}^{k / 4} \frac{1+\varepsilon}{1+\varepsilon-\overline{z_{j} z}}\right| \leq \frac{k(1+\varepsilon)}{4} \sum_{j=1}^{k / 4} \frac{1}{j-1 / 2} \leq C k \log k .
$$

In the same way, we obtain

$$
\left|\sum_{j=3 k / 4}^{k-1} \frac{1+\varepsilon}{1+\varepsilon-\overline{z_{j}} z}\right| \leq C k \log k
$$

whereas $\left|1+\varepsilon-\overline{z_{j}} z\right| \geq C$ for any $j \in[k / 4,3 k / 4]$, so that

$$
\left|\sum_{j=k / 4}^{3 k / 4} \frac{1+\varepsilon}{1+\varepsilon-\overline{z_{j}} z}\right| \leq C k .
$$

Putting this together, we get

$$
|f(z)|=\left|\frac{1}{k} \sum_{j=0}^{k-1} \frac{1+\varepsilon}{1+\varepsilon-\overline{z_{j}} z}\right| \leq C\left(\frac{1}{k \varepsilon}+\log k+1\right) \leq C \exp \left((\log k)^{\beta}\right) .
$$

Finally, it remains to prove (P4). We observe that

$$
f^{\prime}(z)=\frac{1}{k} \sum_{j=0}^{k-1} \frac{(1+\varepsilon) \overline{z_{j}}}{\left(1+\varepsilon-\overline{z_{j}} z\right)^{2}} .
$$

We do not try to get a very precise estimate for $\left|f^{\prime}(z)\right|$ (this is not useful for us). We just observe that $\left|1+\varepsilon-\overline{z_{j}} z\right|^{2} \geq \varepsilon^{2}$ for any $j \in\{0, \ldots, k-1\}$ and any $z \in \mathbb{T}$, so that

$$
\left|f^{\prime}(z)\right| \leq \frac{C}{\varepsilon^{2}}
$$

If we combine this with (P1), we get (P4).

We are almost ready to construct $P$. The next step is to take $h(z)=\log (f(z))$, which defines a holomorphic function in a neighborhood of $\overline{\mathbb{D}}$ by (P1). Moreover, $|\Im m(h(z))| \leq \pi / 2$ for any $z \in \overline{\mathbb{D}}$ and $h(0)=0$. Now, we look at the function $h$ on the boundary of the unit 
disk $\mathbb{D}$, that is we introduce the function $g(x)=h\left(e^{2 i \pi x}\right)$ defined on the circle $\mathbb{T}=\mathbb{R} / \mathbb{Z}$. Properties (P2), (P3) and (P4) can be rewritten as

$$
\begin{aligned}
& \forall x \in I_{k}^{\beta},|g(x)| \geq(\log k)^{\beta}-C \\
& \forall x \in \mathbb{T}, \quad|g(x)| \leq(\log k)^{\beta}+C \\
& \forall x \in \mathbb{T}, \quad\left|g^{\prime}(x)\right| \leq C k^{3} \exp \left(3(\log k)^{\beta}\right) .
\end{aligned}
$$

Now let $n$ be the smallest integer such that $C k^{3} \exp \left(3(\log k)^{\beta}\right) \leq n$. We also have $\left\|g^{\prime}\right\|_{\infty} \leq n$ and we can apply the second part of Lemma 1.6 to the function $\theta(t)=g(t)-g(x)$ when $x \in I_{k}^{\beta}$. Recall that $\|\theta\|_{\infty} \leq 2(\log k)^{\beta}+C$. We get

$$
\left|\sigma_{n} \theta(x)\right| \leq \frac{(\log k)^{\beta}}{2}+C
$$

and we can conclude that

$$
\left|\sigma_{n} g(x)\right| \geq|g(x)|-\left|\sigma_{n} \theta(x)\right| \geq \frac{(\log k)^{\beta}}{2}-C .
$$

We finally set

$$
P=\frac{2}{\pi} e_{n} \sigma_{n}(\Im m g)=\frac{2}{\pi} e_{n} \Im m\left(\sigma_{n} g\right) .
$$

It is straightforward to check that $\|P\|_{\infty} \leq 1$ (recall that $\sigma_{n}$ is a contraction on $\mathscr{C}(\mathbb{T})$ ), and that the Fourier spectrum of $\sigma_{n} g$ is contained in $[1, n-1](\hat{g}(0)=0$ since $h(0)=0)$. Now, the simple algebraic trick exposed at the beginning of the proof shows that

$$
\left|S_{n} P(x)\right|=\left|\frac{1}{\pi} \sigma_{n} g(x)\right|,
$$

so that, for any $x \in I_{k}^{\beta}$,

This leads to

$$
\left|S_{n} P(x)\right| \geq \frac{1}{2 \pi}(\log k)^{\beta}-C
$$

$$
\log \left|S_{n} P(x)\right| \geq \beta \log \log k-C
$$

On the other hand,

$$
\begin{aligned}
\log \log n & \leq \log \left(3 \log k+3(\log k)^{\beta}+\log C\right) \\
& \leq \log \log k+C .
\end{aligned}
$$

Finally,

$$
\frac{\log \log \left|S_{n} P(x)\right|}{\log \log n} \geq \frac{\beta \log \log k-C}{\log \log k+C} \geq(1-\delta) \beta,
$$

provided $k$ has been chosen large enough. Moreover, $n$ can be chosen as large as we want since $n \rightarrow+\infty$ when $k \rightarrow+\infty$.

REMARK 3.4. - The fact that we have to compare $\log \log n$ and $\log \left|S_{n}\right|$ helps us for the previous proof. Even if $n$ and $k$ do not have the same order of growth, this is not apparent when we apply the iterated logarithm.

REMARK 3.5. - During the construction, the integers $k$ and $n$ cannot be chosen independently : they satisfy $n-1 \leq C k^{3} \exp \left(3(\log k)^{\beta}\right) \leq n$ where $C$ is an absolute constant. If we want to construct a polynomial $P$ satisfying the conclusion of Lemma 3.3 with a large value of $n$, we need also to choose a large value of $k$. 


\subsection{The conclusion}

We are now going to prove the full statement of Theorem 1.5. At this point, the situation is less favorable than in the $L^{p}$-case. There, the basic construction done at each step $j$ did not depend on the index of divergence that we would like to get. We had the same function $g_{j}$ which worked for all indices of divergence, and it was the dyadic exponent of $x$ which decided how large $\left|g_{j}(x)\right|$ was. The construction done in Lemma 3.3 is less efficient, because the polynomial $P$ does depend on the expected divergence index $\beta$ (the index $\beta$ is a parameter of the definition of $f$ above). We have to overcome this new difficulty and the solution will be to introduce redundancy in the construction of the $G_{\delta}$-set.

As usual, we start from a sequence $\left(f_{j}\right)_{j \geq 1}$ of polynomials which is dense in $\mathscr{C}(\mathbb{T})$. For convenience, we assume that $\left\|f_{j}\right\|_{\infty} \leq j$ for any $j$ and that the Fourier spectrum of $f_{j}$ is contained in $[-j, j]$. Furthermore, we fix four sequences $\left(\alpha_{l}\right),\left(\beta_{l}\right),\left(\delta_{l}\right)$ and $\left(\varepsilon_{l}\right)$ with values in $(0,1)$ and such that:

$-\left(\beta_{l}\right)$ is dense in $(0,1)$ and $l \mapsto \beta_{l}$ is one to one;

$-\sum_{l} \varepsilon_{l} \leq 1$

$-\left(\delta_{l}\right)$ and $\left(\alpha_{l}\right)$ go to zero.

$-\delta_{l}<1 / 3$.

Let now $j \geq 1$. By induction on $l=1, \ldots, j$, we build sequences $\left(P_{j, l}\right),\left(n_{j, l}\right)$ and $\left(k_{j, l}\right)$ satisfying the conclusions of Lemma 3.3 with $\beta=\beta_{l}, \delta=\delta_{l}$ and $K=j$ (to ensure that $\lim _{j \rightarrow+\infty} k_{j, l}=+\infty$ ) and we will decide how large should $n_{j, l}$ be during the construction. According to Remark 3.5, these constraints on $n_{j, l}$ will determine the values of the $k_{j, l}$. We then set

$$
g_{j}:=f_{j}+\alpha_{j} \sum_{l=1}^{j} \varepsilon_{l} e_{n_{j, l}} P_{j, l}
$$

so that $\left\|g_{j}-f_{j}\right\|_{\infty} \leq \alpha_{j} \sum_{l=1}^{j} \varepsilon_{l}\left\|P_{j, l}\right\|_{\infty} \leq \alpha_{j}$. In particular, the sequence $\left(g_{j}\right)$ remains dense in $\mathscr{C}(\mathbb{T})$.

Recall that the Fourier spectrum of $f_{j}$ is included in $[-j, j]$ and observe that the Fourier spectrum of $e_{n_{j, l}} P_{j, l}$ lies in $\left[n_{j, l}, 3 n_{j, l}-1\right]$. If we suppose that $n_{j, 1}=j+1$ and $n_{j, l+1} \geq 3 n_{j, l}$, we can conclude that the Fourier spectra of $f_{j}, e_{n_{j, 1}} P_{j, 1}, \cdots, e_{n_{j, j}} P_{j, j}$ are disjoint.

Let now $x$ belongs to $I_{k_{j, l}}^{\beta_{l}}$ for some $l \leq j$. Then

$$
\begin{aligned}
\left|S_{2 n_{j, l}} g_{j}(x)\right| & \geq \alpha_{j} \varepsilon_{l}\left|S_{n_{j, l}} P_{j, l}(x)\right|-\alpha_{j} \sum_{m=1}^{l-1} \varepsilon_{m}\left\|P_{j, m}\right\|_{\infty}-j \\
& \geq \alpha_{j} \varepsilon_{l}\left|S_{n_{j, l}} P_{j, l}(x)\right|-\alpha_{j}-j .
\end{aligned}
$$

Because we can choose $n_{j, l}$ as large as we want in the process, we may always assume that the choice that we have made ensures that

$$
\left|S_{2 n_{j, l}} g_{j}(x)\right| \geq \frac{\alpha_{j} \varepsilon_{l}}{2}\left|S_{n_{j, l}} P_{j, l}(x)\right| .
$$


Taking the logarithm, we find

$$
\begin{aligned}
\log \left|S_{2 n_{j, l}} g_{j}(x)\right| & \geq \log \left|S_{n_{j, l}} P_{j, l}(x)\right|+\log \varepsilon_{l}+\log \alpha_{j}-\log 2 \\
& \geq\left(1-\delta_{l}\right) \beta_{l} \log \log \left(n_{j, l}\right)+\log \varepsilon_{l}+\log \alpha_{j}-\log 2 \\
& \geq\left(1-2 \delta_{l}\right) \beta_{l} \log \log \left(2 n_{j, l}\right)
\end{aligned}
$$

provided again that we have chosen $n_{j, l}$ very large.

We then fix $r_{j}>0$ so small that, for any $f \in B\left(g_{j}, r_{j}\right)$ (the balls are related to the norm $\left.\|\cdot\|_{\infty}\right)$, for any $l \leq j$,

$$
\left\|S_{2 n_{j, l}} f-S_{2 n_{j, l}} g_{j}\right\|_{\infty} \leq 1 / 2 .
$$

Observe that for every real number $t \geq 1$, we have $\log (t-1 / 2) \geq \log (t)-\log 2$. For any $x \in I_{k_{j, l}}^{\beta_{l}}$ with $l \leq j$, we get

$$
\begin{aligned}
\log \left|S_{2 n_{j, l}} f(x)\right| & \geq \log \left|S_{2 n_{j, l}} g_{j}(x)\right|-\log 2 \\
& \geq\left(1-2 \delta_{l}\right) \beta_{l} \log \log \left(2 n_{j, l}\right)-\log 2 \\
& \geq\left(1-3 \delta_{l}\right) \beta_{l} \log \log \left(2 n_{j, l}\right)
\end{aligned}
$$

if $n_{j, l}$ are chosen sufficiently large such that $\delta_{l} \beta_{l} \log \log \left(2 n_{j, l}\right) \geq \log 2$.

We finally set

$$
A=\bigcap_{p \in \mathbb{N}} \bigcup_{j \geq p} B\left(g_{j}, r_{j}\right)
$$

and we claim that $A$ is the dense $G_{\delta}$ set we are looking for.

Indeed, let $f$ belong to $A$ and let $\left(j_{p}\right)$ be an increasing sequence of integers such that for every $p \geq 0, f \in B\left(g_{j_{p}}, r_{j_{p}}\right)$. We consider $\beta \in(0,1)$ and choose $p_{0}$ such that

$$
\left\{\beta_{1}, \cdots, \beta_{j_{0}}\right\} \cap(0, \beta) \neq \varnothing .
$$

Such a $p_{0}$ exists since the sequence $\left(\beta_{l}\right)_{l \geq 1}$ is dense in $(0,1)$. For every $p \geq p_{0}$, let $l_{p}$ be chosen in $\left\{1, \cdots, j_{p}\right\}$ such that

$$
\beta-\beta_{l_{p}}=\inf \left\{\beta-\beta_{l} ; l \leq j_{p} \text { and } \beta>\beta_{l}\right\} .
$$

Since the sequence $\left(\beta_{l}\right)$ is dense in $(0,1), \beta_{l_{p}}<\beta$ for $p \geq p_{0}$ and $\beta_{l_{p}} \rightarrow \beta$. Moreover, since $l \mapsto \beta_{l}$ is one to one, it is clear that $l_{p}$ is non decreasing and goes to $+\infty$.

Observe that, for $p \geq p_{0}, I_{k_{j_{p}, l_{p}}}^{\beta} \subset I_{k_{j_{p}, l_{p}}}^{\beta_{l_{p}}}$, so that, for any $x \in I_{k_{j_{p}, l_{p}}}^{\beta}$, setting $N_{p}=2 n_{j_{p}, l_{p}}$,

$$
\log \left|S_{N_{p}} f(x)\right| \geq\left(1-3 \delta_{l_{p}}\right) \beta_{l_{p}} \log \log \left(N_{p}\right) .
$$

In particular, setting $F=\lim \sup _{p} I_{k_{j_{p}, l_{p}}}^{\beta}$, we get that

$$
\limsup _{n \rightarrow+\infty} \frac{\log \left|S_{n} f(x)\right|}{\log \log n} \geq \beta
$$

for any $x \in F$. Now, we can apply Corollary 1.8 with a gauge function $\phi$ satisfying $\phi^{-1}(y)=y \exp \left[-(\log (1 / 2 y))^{\beta}\right]$ to obtain $\mathscr{H}^{\phi}(F)=\infty$.

Observe that if $y=\phi(x)$, then

$$
y=x \exp \left[(\log (1 / 2 y))^{\beta}\right] \quad \text { and } \quad \log x \leq \log y .
$$

It follows that $\phi(x) \leq x \exp \left[(\log (1 / 2 x))^{\beta}\right] \leq \phi_{1,1-\beta}(x)$ and $\mathcal{H}^{\phi_{1,1-\beta}}(F)=+\infty$. 
We now conclude exactly as in the $L^{p}$-case, using Lemma 3.1 to replace Aubry's result. Namely, we set

$$
\begin{aligned}
& F^{1}=\left\{x \in F ; \limsup _{n \rightarrow+\infty} \frac{\log \left|S_{n} f(x)\right|}{\log \log n}=\beta\right\} \\
& F^{2}=\left\{x \in F ; \limsup _{n \rightarrow+\infty} \frac{\log \left|S_{n} f(x)\right|}{\log \log n}>\beta\right\}
\end{aligned}
$$

and we observe that Lemma 3.1 guarantees that $\mathcal{H}^{\phi_{1,1-\beta}}\left(F^{2}\right)=0$. Thus, $\mathcal{H}^{\phi_{1,1-\beta}}\left(F^{1}\right)=+\infty$ and the precised Hausdorff dimension of $F(\beta, f)$, which contains $F^{1}$, is at least $(1,1-\beta)$. By Lemma 3.1, it is exactly $(1,1-\beta)$.

REMARK 3.6. - It is amazing that, with our method, it is easier to prove Theorem 1.5 than to directly prove that the Hausdorff dimension of the sets $F(\beta, f)$ are all equal to one. Indeed, to ensure that the sets $F(\beta, f)$ are big, we need to know that the sets $\mathcal{F}\left(\beta^{\prime}, f\right)$ are small for $\beta^{\prime}>\beta$. This cannot be done if we stay within the notion of Hausdorff dimension.

REMARK 3.7. - The method developed above allows us to construct a "concrete function" that satisfies the conclusion of Theorem 1.5. More precisely, it suffices to consider

$$
g=\sum_{j=1}^{+\infty} \frac{1}{j^{2}} \sum_{l=1}^{j} \varepsilon_{l} e_{n_{j, l}} P_{j, l}
$$

with the constraint $3 n_{j, j}<n_{j+1,1}$ to ensure that the blocks $\sum_{l=1}^{j} \varepsilon_{l} e_{n_{j, l}} P_{j, l}$ have disjoint Fourier spectra. Such a function is some kind of saturating function in the continuous case.

Remark 3.8. - Refining the method developed here, in [2] we have been able to prove the following result: for any sequence $\left(\varepsilon_{n}\right)$ decreasing to 0 , quasi surely in $\mathscr{C}(\mathbb{T})$, the set of points $x \in \mathbb{T}$ such that $\lim \sup _{n \rightarrow+\infty} S_{n} f(x) /\left(\varepsilon_{n} \log n\right)=+\infty$ has Hausdorff dimension 1.

REMARK 3.9. - If $f \in L^{p}(\mathbb{T})$, we always have $\operatorname{dim}_{\mathcal{H}}(E(0, f))=1$. We could analyze this set more finely by introducing again the family of sets $F(\beta, f)$, allowing now $\beta$ to live in $(0,+\infty)$. In this $L^{p}$ context, we do not know the corresponding gauges.

\section{Acknowledgments}

We thank the referees for their careful reading of the paper and their constructive remarks. We also thank A. Durand for fruitful discussions.

\section{REFERENCES}

[1] J.-M. Aubry, On the rate of pointwise divergence of Fourier and wavelet series in $L^{p}$, J. Approx. Theory 138 (2006), 97-111.

[2] F. Bayart, Y. Heurteaux, Multifractal analysis of the divergence of Fourier series: The extreme cases, preprint arXiv:1110.5478.

[3] V. Beresnevich, S. Velani, A mass transference principle and the Duffin-Schaeffer conjecture for Hausdorff measures, Ann. of Math. 164 (2006), 971-992. 
[4] M. M. Dodson, M. V. Melián, D. Pestana, S. L. Velani, Patterson measure and ubiquity, Ann. Acad. Sci. Fenn. Ser. A I Math. 20 (1995), 37-60.

[5] K. FAlCONER, Fractal geometry. Mathematic foundations and applications, second ed., John Wiley \& Sons Inc., 2003.

[6] S. Jaffard, On lacunary wavelet series, Ann. Appl. Probab. 10 (2000), 313-329.

[7] S. Jaffard, On the Frisch-Parisi conjecture, J. Math. Pures Appl. 79 (2000), 525-552.

[8] J.-P. Kahane, Y. Katznelson, Sur les ensembles de divergence des séries trigonométriques, Studia Math. 26 (1966), 305-306.

[9] J.-P. Kahane, R. Salem, Ensembles parfaits et séries trigonométriques, Actualités Sci. Indust. 1301, Hermann, 1963.

[10] Y. Katznelson, An introduction to harmonic analysis, John Wiley \& Sons Inc., 1968.

[11] P. Mattila, Geometry of sets and measures in Euclidean spaces, Cambridge Studies in Advanced Math. 44, Cambridge Univ. Press, 1995.

[12] P. Mörters, Y. Peres, Brownian motion, Cambridge Series in Statistical and Probabilistic Mathematics, Cambridge Univ. Press, 2010.

[13] J. Arias de Reyna, Pointwise convergence of Fourier series, Lecture Notes in Math. 1785, Springer, 2002.

[14] A. Zygmund, Trigonometric series, 3rd ed., Cambridge Univ. Press, 2003.

(Manuscrit reçu le 16 mars 2011;

accepté, après révision, le 12 juin 2012.)

\author{
Frédéric BAYART \\ Clermont Université, Université Blaise Pascal \\ Laboratoire de Mathématiques \\ BP 10448 \\ 63000 Clermont-Ferrand, France \\ et \\ CNRS, UMR 6620 \\ Laboratoire de Mathématiques \\ 63177 Aubière Cedex, France \\ E-mail: Frederic.Bayart@math.univ-bpclermont.fr \\ Yanick Heurteaux \\ Clermont Université, Université Blaise Pascal \\ Laboratoire de Mathématiques \\ BP 10448 \\ 63000 Clermont-Ferrand, France \\ et \\ CNRS, UMR 6620 \\ Laboratoire de Mathématiques \\ 63177 Aubière Cedex, France \\ E-mail: Yanick.Heurteaux@math.univ-bpclermont.fr
}

$4^{\text {e }}$ SÉRIE - TOME $45-2012-$ N $^{\circ} 6$ 